\title{
Pratiques
}

Linguistique, littérature, didactique

\section{Didactique du lexique : état des lieux et nouvelles orientations}

Francis Grossmann

\section{(2) OpenEdition}

Journals

Édition électronique

URL : http://journals.openedition.org/pratiques/1732

DOI : 10.4000/pratiques. 1732

ISSN : 2425-2042

Éditeur

Centre de recherche sur les médiations (CREM)

Édition imprimée

Date de publication : 15 juin 2011

Pagination : 163-183

Référence électronique

Francis Grossmann, "Didactique du lexique : état des lieux et nouvelles orientations », Pratiques [En ligne], 149-150 | 2011, mis en ligne le 17 juin 2014, consulté le 19 avril 2019. URL : http:// journals.openedition.org/pratiques/1732 ; DOI : 10.4000/pratiques.1732

(c) Tous droits réservés 


\title{
Didactique du lexique : état des lieux et nouvelles orientations
}

\author{
Francis Grossmann \\ Université Stendhal, Grenoble 3, Laboratoire LIDILEM \\ (Linguistique et didactique des langues étrangères et \\ maternelles), E.A. 609
}

Par un retour de balancier classique, la didactique du lexique ${ }^{(1)}$ après avoir subi une éclipse dans les années 1980-2000, davantage centrées sur la lecture et l'écriture, revient depuis une dizaine d'années au premier plan. Le paysage a cependant été profondément modifié, tant en raison des évolutions des théories de références, en lexicologie et en acquisition, qu'en raison des modifications qui se sont opérées dans les approches didactiques elles-mêmes. Citons en vrac quelques uns des apports « externes » qui ont nourri le terreau de la réflexion didactique ces dernières années : en acquisition, les recherches sur l'accès lexical et l'amorçage sémantique ; en linguistique et en psychologie, le renouvellement des approches sémantiques, les grammaires cognitives et les recherches sur la catégorisation (théories du prototype) ; l'influence toujours très forte en didactique des linguistiques du discours et de l'énonciation; en sociolinguistique la poursuite des travaux sur la variété des usages ; en lexicologie, l'impact des recherches sur la phraséologie, le figement dans des cadres divers (Théorie SensTexte, grammaires de construction...). Comme il est impossible de dresser un état des lieux exhaustif des directions de recherche qui se sont développées depuis une vingtaine d'années, j'assume la part de subjectivité inévitable des choix effectués dans cette présentation. C'est que la problématique lexicale me semble d'abord devoir être traitée comme une interface : elle établit des ponts avec la catégorisation et la cognition, avec les discours, et donc avec l'énonciation, ainsi qu'avec la syntaxe bien évidemment. Ce sont donc ces aspects qui serviront de portes d'entrée à mon propos, avec une attention particulière à un problème qui concerne directement l'apprentissage : dans quelle mesure et comment con-

(1) Nous utiliserons préférentiellement le terme «lexique » dans cette contribution. L'opposition classique entre le lexique comme appartenant au système de la langue et le vocabulaire, comme représentant la part du lexique instanciée par un individu ou un groupe social se trouve dans les faits biaisée : comme le remarque Petit (2000), le terme vocabulaire est utilisé préférentiellement à l'école primaire et au collège, tandis que lexique est davantage mobilisé dans 1'enseignement supérieur. 
vient-il de prendre en compte la phraséologie et les collocations ? Comment articuler les démarches portant sur le lexique avec le travail sur les genres et les discours, et en particulier avec les genres spécifiques que rencontrent élèves et étudiants au cours de leurs études? Ma contribution s'organisera en trois parties, la première reviendra sur les débats concernant la démarche à mettre en œuvre, et sur la question des répertoires; la seconde portera sur le développement d'une meilleure compréhension du système lexical ; la troisième enfin traitera des approches « intégratives », reliant l'enseignement lexical aux activités d'écriture et de lecture.

\section{Aspects quantitatifs et qualitatifs de l'apprentissage lexical}

\subsection{Apprentissage « incident » et « explicite »}

On a l'habitude d'opposer deux démarches en ce qui concerne l'enseignement du lexique : l'apprentissage "incident », qui ménage des temps d'explication lexicale au sein d'activités de lecture ou d'écriture, et l'apprentissage " explicite », qui prévoit une progression dans 1'apprentissage, appuyée sur 1'appropriation de notions métalexicales (ex. champ sémantique, polysémie, synonyme, antonyme...). Dans la pratique, cette distinction s'avère parfois artificielle, puisque même pour l'apprentissage incident sont généralement mobilisés des termes métalinguistiques permettant de caractériser le lexique qui est étudié. La différence vient du fait que dans le premier cas, ces notions font l'objet d'un enseignement systématique correspondant à des activités spécifiques, tandis que dans la seconde les définitions des termes sont amenées au fur et à mesure des besoins, au cours des activités d'expression et de communication. Par rapport à ces deux grandes tendances, celle qui prône l'apprentissage incident, occasionnel, et celle qui privilégie un apprentissage structuré » (cf. par ex. Tremblay, 2004, 2009) visant une meilleure analyse du lexique, l'enseignant de base ne peut que ressentir une certaine frustration : il sait bien, quant à lui que, pour une part au moins, un des problèmes rencontrés par les élèves réside dans la maîtrise de répertoires lexicaux diversifiés et que l'hétérogénéité sociale conduit à des différences importantes, ce qui a des répercussions notamment dans le domaine de la lecture et de l'écriture de textes. Pour sortir de cette contradiction entre le point de vue des chercheurs et celui des praticiens, il paraît utile de proposer une définition véritablement didactique de la compétence lexicale : celle-ci doit prendre en compte des aspects quantitatifs (on ne peut balayer d'un revers de main la question de l'étendue du vocabulaire passif/actif) et de la constitution de répertoires adaptés ; mais elle inclut également des aspects qualitatifs, liés au développement de compétences métalinguistiques; cette compétence inclut aussi la capacité à faire des hypothèses sur le sens d'un mot inconnu, à partir d'éléments morphologiques, phrastiques ou textuels et à construire et affiner progressivement le sens des unités lexicales, en distinguant leurs différentes acceptions mais aussi en les intégrant dans des réseaux sémantiques.

Un certain consensus s'établit donc aujourd'hui chez les didacticiens qui s'intéressent à ces questions pour penser qu'il faut à la fois établir une progression des notions de base nécessaires à la compréhension du système lexical et mieux réfléchir aux formes que peut prendre l'apprentissage "incident », à partir de genres discursifs variés. En effet, les deux aspects sont complémentaires et nul- 
lement contradictoires. Picoche (2004, p. 221) illustre bien le caractère indispensable d'une approche autonome en soulignant que jamais un texte littéraire - cela est vrai d'ailleurs pour tout type de texte - ne permet l'organisation d'un réseau sémantique complet et organisé, c'est-à-dire comportant les lexies associées par des liens sémantiques et/ou morphologiques, ainsi qu'à travers la structure actancielle. Ce constat n'implique pas qu'il ne faut pas mettre chacun de ces associés «en contexte » ou en situation, c'est même indispensable. De la même façon, Sonia Branca-Rosoff et al. (2008, p. 266) soulignent que « c'est en installant des moments réguliers d'observation réfléchie de la langue, bien déconnectés du flux de la communication qu'on peut espérer développer une conscience métalinguistique chez les élèves ». Et cela n'empêche pas de mobiliser les connaissances acquises pour analyser le lexique rencontré au cours des lectures.

Lorsque le professeur ne se contente pas d'expliquer les mots au fil des lectures, comment procède-t-il pour " travailler » le lexique ? Une des premières questions qui se pose est la sélection des mots ou expressions à étudier ${ }^{(2)}$. La sélection du lexique d'étude est tributaire des représentations que se fait l'enseignant des difficultés que rencontrent les élèves dans le domaine lexical, à différents niveaux, au cours des activités d'expression et de communication, ainsi que des genres mobilisés. Elle peut aussi prétendre à plus de scientificité, en voulant fonder la progression sur des critères objectifs, liés en particulier à la fréquence d'usage des mots rencontrés dans les textes.

\section{2. « Mot juste ", « mots difficiles », reformulation et ajustement lexical}

Un exercice classique, à l'école primaire et au collège, consiste à proposer des énoncés aux élèves, dans lesquels on leur demande de «trouver le mot juste », les élèves étant encouragés à éviter les termes passe-partout. La notion de mot juste reste très tenace parce qu'ancrée dans le mythe littéraire d'une expression parfaite, qui permettrait de faire coïncider langage et réalité. Dès 1975, S. Delesalle avait analysé finement, à travers les manuels d'expression, les apories auxquelles conduisaient les exercices visant à retrouver les « mots justes » dans des énoncés préfabriqués. Une thèse récente (Charles, 2008) qui adopte par ailleurs un point de vue plutôt positif sur la notion, montre la manière dont elle est couplée avec la notion complémentaire d'enrichissement lexical ${ }^{(3)}$ : à partir du moment où l'on considère comme indispensable l'enrichissement lexical, il convient en effet de réfléchir à la sélection des termes appropriés au bon fonctionnement de l'expression (d'où également le succès de la notion de registre). Ce qui est mis en évidence dans la thèse, c'est qu'un bond qualitatif est souvent demandé à l'élève, la connaissance du sens du mot étant à elle seule censée autoriser un emploi approprié. Or l'absence d'intégration des dimensions lexico-syntaxiques et discursives ne permet justement pas de fournir aux élèves les outils nécessaires pour l'emploi pertinent des mots travaillés. C'est d'autant plus para-

(2) Difficile d'éviter le mot «mot». Comme le remarque Petit (2000) «1'archétype du mot habite la conscience du didacticien comme celle du linguiste ». Il est nécessaire cependant d'éviter toute confusion entre le terme «mot», qui renvoie de manière pré-scientifique à l'unité lexicale, et le mot-forme, qui est l'unité d'analyse morphologique.

(3) Paveau (2000), dans une analyse critique serrée de la notion même de richesse lexicale montre également à quel point celle-ci trouve ses fondement dans la tradition française du «mot juste». 
doxal que des normes d'emploi conditionnent par ailleurs la sélection du « mot juste » telle qu'elle est préconisée, notamment l'évitement des fameuses « répétitions ». Le recours à des listes du type dites, ne dites pas, qui semblent fournir un prêt-à-écrire exclut de fait une réflexion qui ferait de l'examen du contexte - incluant les différents systèmes de normes, y compris celui du genre - le préalable à la recherche d'une expression lexicale pertinente.

La notion de «mot juste » a cependant récemment donné lieu à des recherches plus novatrices, par exemple en ce qui concerne le tâtonnement qu'entraîne la recherche du « mot juste » à l'oral (voir Cappeau, 2008, qui étudie les ajustements lexicaux auxquels procèdent les locuteurs au cours de l'interaction, mettant en œuvre différentes formulations, concurrentes ou complémentaires, avec des éléments de retouche, pour approcher de plus près ce qu'ils veulent dire). L'apprentissage lexical a partie liée avec la reformulation, sous toutes ses formes, l'idée d'ajustement réciproque permettant d'introduire également la dimension dialogique, plutôt que d'imposer comme le fait la notion de «mot juste », l'idée d'une norme abstraite, valable quelle que soit le contexte discursif (pour une prise en compte des interactions langagières et de la reformulation dans le développement de la compétence lexicale, voir Guernier, 2005, Martinot, 2006 ; LepoireDuc, 2008).

Pour se résumer, si l'on entend par « recherche du mot juste» le souci de la précision de l'expression et de son exactitude, il va de soi que ce programme fait partie de plein droit de toute didactique digne de ce nom. Encore faut-il s'entendre sur les conditions qui le permettent : or celles-ci relèvent avant tout de l'ordre de propriétés combinatoires ainsi que de normes discursives : qu'il s'agisse de contraintes liées au genre, ou, sur un plan plus microlinguistique, de contraintes d'associations lexicales. Le choix des mots par l'élève ne peut donc être uniquement envisagé sur un plan paradigmatique, mais prend en compte également les propriétés syntagmatiques, ce qui suppose qu' on lui fournisse les outils théoriques et pratiques pour effectuer ces choix. Le mot juste est souvent considéré comme plus « précis » que celui qui est employé effectivement; l'idée de « richesse lexicale» se fonde sur les représentations sociales des enseignants (et audelà, de toute la société), stigmatisant le "vocabulaire pauvre » de certains élèves, sans pour autant qu'un programme d'action efficace ne soit déclenché pour pallier ces manques, réels ou prétendus, ce qui supposerait une évaluation objective des difficultés. Plane (2004) à travers une expérimentation demandant à des enseignants de prévoir des annotations pour les «mots difficiles » dans un texte de vulgarisation scientifique, montre bien qu'il est difficile d'anticiper les difficultés réelles des élèves : la faible fréquence suffit généralement à faire considérer le mot comme difficile; 1'encyclopédisme est privilégié ; les enseignants ont tendance à ne pas définir des mots qui appartiennent à leur répertoire lexical professionnel ; certaines expressions qui leur semblent parfaitement compréhensibles peuvent cependant présenter de réelles difficultés.

\subsection{Construire un «vocabulaire de base » pour asseoir la compétence lexicale}

Comme le rappelle Vigner (1997), il existe un décalage important entre ce qu'il est possible de faire dans le cadre d'un apprentissage systématique (quelques centaines de mots tout au plus) et le développement lexical, tel qu'il s'ef- 
fectue au cours de l'acquisition. Partant du constat de l'importance du volume du répertoire lexical de l'individu (environ 40.000 mots) et de l'extraordinaire vitesse de croissance de ce répertoire (au minimum 3000 mots par an durant la période de la scolarité) on a parfois conclu (voir par exemple W. Nagy et P. Herman, 1987) au peu d'intérêt et d'efficacité d'un apprentissage délibéré qui ne pourrait porter que sur 3 ou 400 mots par an. La situation est évidemment différente en L2, où la nécessité d'un apprentissage systématique du lexique est beaucoup plus évidente, bien que délicate à élaborer.

A partir de ce constat, une voie a été explorée dans le passé : elle consiste à partir de la fréquence (et, complémentairement, de la dispersion) pour définir un « vocabulaire de base $»$. Benigno ${ }^{(4)}$ rappelle que la fréquence est le critère essentiel des travaux qui ont donné origine aux listes du vocabulaire de base dans différentes langues : pour l'anglais le « Basic English» (Ogden, 1934), pour le français le «Français Fondamental» (Gougenheim et al., 1958/1964), pour l'italien le « Vocabolario di base » (De Mauro, 1983). La dénomination « vocabulaire de base » semble aujourd'hui désuète ; il y a cependant des arguments empiriques forts, comme le signale Benigno, pour maintenir - en la renouvelant - une telle approche : d'abord les données acquisitionnistes (Tomasello, 2003) montrent que les mots les plus fréquents sont acquis les premiers, et «par blocs», au sein de structures lexico-syntaxiques à fonction pragmatique ; les données neurolinguistiques vont également dans le même sens, et signalent une corrélation forte entre la fréquence d'occurrence d'un item et sa possible activation dans le lexique mental. On peut penser que cette corrélation s'explique par le fait que les mots les plus fréquents représentent de bons prototypes pour l'apprentissage lexical, en ce qu'ils fournissent des exemplaires à partir desquels l'enfant, dans sa langue maternelle, ou l'apprenant, lorsqu'il débute dans une langue étrangère peuvent plus facilement construire le système lexical. L'idée du vocabulaire de base était d'établir des priorités, de manière à sérier les difficultés et à programmer " scientifiquement » l'apprentissage. Elle peut se comprendre aujourd'hui plutôt comme une manière d'ajuster l'apprentissage sur l'acquisition ${ }^{(5)}$, en se souvenant cependant qu'il n'y a pas parfaite isomorphie entre les deux aspects, dans la mesure où l'exposition au lexique nouveau précède largement son appropriation.

Matheys (1992) a fait un bilan critique des apports des pionniers qui se sont appuyés sur la statistique lexicale en combinant les critères de fréquence et de dispersion pour construire l'apprentissage lexical, en relevant trois aspects principaux. Derrière la notion de vocabulaire fondamental, il y a d'abord l'idéal d'une culture commune, le vocabulaire fondamental représentant à la fois la langue de la démocratie, et celle des « bons auteurs » :

« il est remarquable de constater que ce sont les meilleurs auteurs qui font le plus large usage du vocabulaire fondamental. Ils disent beaucoup avec peu de moyens. Ce sont des classiques. Les auteurs de second ordre masquent souvent l'indigence de leur pensée sous l'abondance de mots rares, absents du vocabulaire fondamental. La soi-disant richesse du vocabulaire est souvent pédanterie. » (Ters et al., cité par Matheys 1992, p. 109).

(4) Thèse en cours sur le lexique de base en français et en italien.

(5) Voir Duvignau (2005) pour des propositions au niveau maternelle. 
Ensuite, derrière le lexique, c'est l'orthographe et son évaluation qui est souvent prioritairement visées, comme l'illustrent bien les travaux de Ters, Mayer et Reichenbach (1964). Il s'agit en définitive, dit Matheys, de classer, de normaliser. Pour finir, l'approche semble disqualifiée par le renversement didactique qui s'est produit dans les années 80 : à partir du moment où l'on considère - y compris dans les instructions officielles des différents pays francophones, que le point de départ du développement des compétences linguistiques réside dans la capacité à communiquer :

«Alors que l'enseignement traditionnel du français préconise l'apprentissage du vocabulaire pour pouvoir communiquer, les directives actuelles renversent la formule en proclamant que c'est en communiquant qu'on apprend, ce qui relativise la problématique des vocabulaires de base puisqu'on part des activités des élèves et non d'un ensemble prédéterminé de mots. » (ouvr. cit. p. 110).

C'est sans doute ce qui explique que dans l'espace francophone, peu de chercheurs aient repris le flambeau de Gougenheim. Quelques tentatives isolées témoignent régulièrement de l'intérêt que peut représenter cette problématique. Etienne Brunet, à partir de la base Frantext, a fait la liste de 1500 mots, les plus fréquents de la langue française (en ligne sur le site Eduscol). Cette liste veut « rendre compte de la langue que lisent les élèves francophones », mais l'on voit bien qu'aucun accompagnement didactique n'est proposé, à l'inverse des travaux réalisés à la période précédente. Dans une perspective voisine et avec une souci beaucoup plus clair des exploitations possibles, la base lexicale MANULEX (Lété, Sprenger-Charolles, \& Colé, 2004), disponible sur le web, fournit les fréquences d'occurrences de 23.900 lemmes et 48.900 formes orthographiques extraits d'un corpus de 54 manuels scolaires de lecture à différents niveaux d'enseignement de l'école primaire (CP, CE1, et cycle 3). Il s'agit ici plutôt d'évaluer le type de lexique que peuvent rencontrer les élèves lors de l'apprentissage scolaire, notamment lors de l'acquisition de la lecture. Pour l'enseignement du vocabulaire, les enseignants du primaire peuvent extraire de ces tables de fréquence des listes de mots adaptées aux enfants de cycles 2 et 3.

On le voit, une enquête plus large, portant sur le lexique en usage aujourd'hui serait entièrement à reprendre pour le français. Ce qui était révolutionnaire dans le projet du «français fondamental », c'était d'intégrer le lexique des élèves euxmêmes, à travers l'oral, et les textes de copies. Ce programme reste d'actualité : il est important aujourd'hui comme le soulignent Branca-Rosoff et al. (2008, p. 253) de mener des enquêtes empiriques sur le lexique utilisé par les élèves dans différentes situations. Cette équipe en fournit un exemple à travers les résultats d'une enquête menée dans le cadre d'un appel d'offre de la DGLF, visant à rendre compte du "lexique-grammaire» des sensations d'élèves de 7 à 9 ans, à travers une vingtaine d'heures de transcription orale. D'une manière générale, le vocabulaire des sensations mobilisé dans l'oral courant est relativement réduit, surtout par rapport à la langue littéraire. Lorsqu'il l'est, c'est principalement dans des buts d'évaluation (essentiellement l'opposition entre plaisant et déplaisant). De tels constats permettent d'orienter utilement le type de travail qui peut être mené en classe. Nous revenons en section 2.3. sur la question de la polysémie, qui doit être intégrée à toute réflexion sur ce thème, la prise en compte de nouvelles acceptions d'un polysème représentant un élément essentiel pour l'apprentissage. 
Plutôt qu'un «vocabulaire fondamental », c'est donc un ensemble de répertoires qu'il faudrait pouvoir construire aujourd'hui, des lexiques des genres et des situations, intégrant la diversité et la variation, et les jeux de langage relevant eux-mêmes de genres de discours diversifiés, nécessitant parfois un lexique plus spécialisé, " ouvrant les portes de champs de savoirs indispensables à une scolarité réussie ou plus largement à une meilleure compréhension du monde (Leclaire-Halté et Rondelli, 2010, p. 139). On a vu se développer par exemple, des enquêtes portant sur le lexique de l'écrit universitaire et ses difficultés, prenant en compte les discours « experts » et les productions réalisées par des étudiants néophytes ou les jeunes chercheurs ( $c f$. Rinck, 2006) qui montrent également dans quelles directions on peut se diriger. De nombreux chantiers de ce type restent à ouvrir.

\section{Développer la compréhension du système}

L'idée que le développement des compétences lexicales passe par une meilleure connaissance du fonctionnement linguistique - et en particulier de son module lexical - ne va pas de soi, en ce qui concerne la langue première tout au moins : sauf à l'écrit - ou dans certains genres exigeant un oral très surveillé -, lorsque nous sommes amenés à sélectionner les mots avec davantage d'attention, nous n'avons pas à les analyser en détail, ni à les rapprocher les uns des autres pour pouvoir communiquer. On considère généralement que l'accès lexical est automatisé, sauf lors d'activités spécifiques (par exemple lors de l'apprentissage de la lecture) ou en cas de « pannes » momentanées ou liées à des maladies dégénératives. Remarquons que le débat, de fait, est double, puisque la question du caractère systématique $v s$ informel de l'apprentissage lexical ne se superpose pas à celle qui porte sur la place de la conscience linguistique (ou métalinguistique) dans l'apprentissage. Dans la démarche que nous avons évoquée concernant les travaux sur la fréquence, l'approche se voulait on ne peut plus systématique et progressive. En revanche, elle se fondait essentiellement sur des listes de mots, et non sur l'appropriation de structures oufonctions lexicales, c'est-à-dire prenant en compte la combinatoire sémantique et syntaxique. Si la capacité à analyser la manière dont fonctionne le système lexical n'est pas liée directement aux compétences communicatives les plus immédiates, elle semble de plus en plus requise, au fur et à mesure du développement de la scolarité, notamment dans les différents genres écrits scolaires puis universitaires. La définition d'un enseignement structuré du lexique est passée par des chemins variés, dont je me contenterai de fournir ici quelques exemples significatifs, à la fois pour rafrâ̂chir notre mémoire collective et pour évaluer leur intérêt actuel.

\subsection{Les exercices portant sur la morphologie}

Les «transformations » ont fourni la matière d'exercices qui peuvent sembler aujourd'hui dépassés, puisqu'ils s'inscrivaient alors dans le contexte des grammaires structurales et transformationnelles ${ }^{(6)}$. Leur conception charrie toute la

(6) On se reportera sur ce point à l'analyse critique effectuée par Cottez (1977) de certains exercices de transformation proposés par des manuels dans les années 70 : "on remet une décoration aux sauveteurs $» \rightarrow$ la remise d'une décoration $»$. Or, remise, seul dérivé de re- 
tradition des exercices structuraux, et pose également, un problème complexe, celui de l'intégration de la dimension morphologique, sur laquelle on fonde partiellement la compétence lexicale. Ce qui a vieilli, en définitive, c'est l'idée du montage d'automatismes effectué sans travail préalable sur les conditions spécifiant le type de dérivé mobilisé dans les transformations. Un travail beaucoup plus fin est nécessaire, si l'on veut se placer dans cette perspective, afin d'éviter les approximations et les caricatures. Faire réfléchir les élèves aux « transformations ", c'est-à-dire en définitive au système de dérivation d'une langue implique d'appréhender le fait lexical dans toute sa complexité, c'est à dire la diversité des acceptions et des dérivations qu'elles autorisent, en prenant en compte également les constructions syntaxiques et la caractérisation des actants, et en veillant à bien distinguer les différentes lexies rassemblées sous un même vocable ${ }^{(7)}$. C'est donc bien d'une activité réflexive qu'il s'agit, qui implique un questionnement, et ne peut se limiter à une mécanique.

Le même type de remarque peut être formulé à propos des exercices de combinaison morphologique, visant à retrouver, voire à inventer, des mots construits. Il reste certes utile de faire jouer les élèves en montrant comment sont « fabriqués » les mots construits (pour des propositions, voir Calaque, 2002). Comme le remarque Lüdi (1997), on postule généralement aujourd'hui « une sous-compétence lexicale double, composée d'une part de listes de mots dans une mémoire lexicale et d'autre part de règles lexicales ", ce qui permet de comprendre comment un locuteur-auditeur particulier peut produire et comprendre des unités construites nouvelles ou inconnues de lui. Mais la notion de «transparence », souvent invoquée, n'est pas toujours... transparente : il reste nécessaire d'interroger les contraintes réciproques imposées par les affixes et par la base. Ainsi, un préfixe comme pré ne peut se combiner avec n'importe quel type de lexème: il exige une unité temporellement bornée, avec un procès comportant un début, et une fin (Amiot, 1997, Petit, 2000), et le fait même d'adjoindre ce préfixe impose une lecture événementielle à des noms que l'intuition n'aurait pas considéré comme tels ( $c f$. pré-cartilage : "cartilage embryonnaire »). Le mécano, quand il peut se jouer, ne se joue pas n'importe comment, et la construction du sens des mots complexes mérite un examen attentif : selon le degré de diagrammaticité (Apothéloz, 2002 :49) d'un mot construit, le locuteur-auditeur évalue sa conformité par rapport à un type idéal : un fort degré de diagrammaticité ${ }^{(8)}$ d'un mot se caractérise pour les usagers par une bonne lisibilité de sa structure interne ; il semble donc préférable de partir des lexies complexes, qui ont un degré de diagrammaticité suffisant pour l'observation. Des outils comme le Robert Brio (Rey-Debove, 2004) et pour les collocations le Dictionnaire des combinaisons de mots de Le Fur (2007) ainsi que le Lexique actif du français (LAF) de

mettre ne permet pas de nominaliser «ne plus remettre les pieds chez quelqu'un », "je vous remets », "remettre du sel ", "je m'en remets à vous »: on néglige ici le fait que la nominalisation ne peut se faire que sur certains emplois de la base, et à partir d'une désambiguïsation.

(7) Nous reprenons ici la terminologie de la lexicologie combinatoire, voir Mel'čuk et al. (1995).

(8) La diagrammaticité d'après Apothéloz résulte de trois facteurs : la compositionnalité (arrosoir, rasoir, fermoir, déchaussoir, démêloir... mais réservoir, saloir?) ; la productivité des morphèmes, c'est-à-dire le nombre de lexèmes qu'ils permettent de décrire par rapport à la quantité de lexèmes nouveaux qu'ils peuvent produire; enfin la transparence formelle. 
Mel'čuk et Polguère (2007) fournissent des éléments d'analyse utiles, dès la fin de la scolarité primaire et le niveau du collège.

D'une manière plus générale, les exercices consistant à mettre à jour des « $f a$ milles de mot ", hésitent encore souvent, dans les classes où on les pratique encore, entre une définition historique et une conception plus nettement sémantique, rassemblant uniquement les apparentés morphologiques qui conservent un noyau de sens commun. Les deux exercices peuvent avoir leur intérêt (bien que le premier soit plutôt réservé aux niveaux les plus élevés), mais il est nécessaire de les distinguer nettement. Mel'čuk et al. (1995) proposent de leur côté une conception résolument sémantique, puisque la notion de "dérivés sémantiques » intègre également chez eux des lexies qui n'ont pas d'apparentement morphologique.

\subsection{L’analyse sémique : une vieille dame indigne ?}

Cet exercice populaire en son temps, souvent critiqué ou rejeté aujourd'hui (voir par exemple Leeman, 2000) n'est certes pas une panacée, mais il permet de comparer la manière dont s'organise, du point de vue sémantique, un système lexical, en langue maternelle ou dans différentes langues; il conserve une vertu explicative indéniable, pour peu qu'il soit manié avec suffisamment de précaution ; ainsi, pour éviter de se perdre (et les élèves avec !) mieux vaut cantonner l'analyse à des micro-systèmes linguistiques, permettant par exemple d'opposer les nuances de sens de quelques lexies. D'après ses détracteurs, l'analyse sémique serait davantage une analyse du monde qu'une analyse du fonctionnement des mots. Cependant ce constat est à relativiser, si l'on prend soin de fonder l'analyse sur des propriétés linguistiques : par exemple, le fait que l'on s'assoie « dans » un fauteuil mais « sur » une chaise, témoigne bien de l'existence d'un sème « enveloppant » permettant de distinguer fauteuil et chaise, sème qui se révèle plus clairement distinctif que le traditionnel « avec bras » (il existe des fauteuils modernes en plastique, sans bras). Il n'est certes pas toujours aisé de trouver des caractéristiques linguistiques pertinentes, mais l'idée qui doit guider le pédagogue, c'est bien le fait que les différences sémantiques se manifestent dans des différences d'emploi, syntaxiques et/ou discursives. D'autre part, dans une perspective d'éveil aux langues, l'analyse sémique peut aider à mettre en évidence les logiques (avec les lacunes lexicales) des systèmes de dénomination d'une langue à l'autre. Cependant, il est vrai que le modèle différentialiste de l'analyse sémique se révèle souvent inadéquat. Comme le remarque Petit (2000, p. 2), qui se place dans une perspective référentielle, les couples tube et tuyau ou immeuble et maison restent réfractaires à une approche systémique, et demandent d'autres types d'approche pour être étudiés ${ }^{(9)}$. Faut-il pour autant jeter le bébé avec l'eau du bain ? Intuitivement, tout usager de la langue française sait que le mot nostalgie renvoie à une forme de /tristesse//liée à des souvenirs/, et que c'est bien l'association aux souvenirs qui en fait une tristesse pas comme les autres. Lorsqu' on construit des définitions - exercice qui conserve ses vertus pédagogiques - on procède souvent à une analyse sémique, que l'on le veuille ou non.

(9) Les apports des sémantiques du prototype peuvent inspirer des travaux pratiques sur les représentations lexicales des élèves, et certaines formes de définition. 


\subsection{L’analyse des polysèmes}

L'approche d'inspiration guillaumienne proposée par Jacqueline Picoche se fonde sur la polysémie et le potentiel symbolique des mots :

« le point de départ d'un enseignement systématique du vocabulaire me paraît [...] résider dans l'étude des mots très peu spécifiques, très peu connotés, à la fois hyperonymiques et très polysémiques, qui apparaissent en tête des différentes fréquences. C'est là que se trouvent les primitifs sémantiques à partir desquels les hyponymes sont construits et c'est dans la structure de leurs polysémies qu'apparaissent les mécanismes conceptuels de base propres à la langue française comme à toute autre langue maternelle. » (Picoche, 1992:19)

Cette démarche peut sembler aller à l'encontre du fait, que dans la pratique, ce qui semble manquer aux élèves, ce ne sont pas ces mots à très grande fréquence, mais bien des termes plus spécialisés, et leur usage, appartenant à différents domaines de connaissance, à différentes disciplines, ou encore à la langue écrite littéraire : autrement dit, tout ce qui ne compose pas leur vernaculaire.

C'est ce que remarque Vigner (1997 : 61) pour qui cette approche tend à transférer dans la classe la démarche du chercheur, mais [...] ne résout qu'improprement les problèmes de développement du répertoire lexical ». Il convient cependant de faire deux observations :

- d'une part, bien que Jacqueline Picoche ne pose pas exactement le débat en ces termes, elle plaide vigoureusement pour une approche réflexive du fait lexical, qui nécessite un appui théorique et méthodologique spécifique ; - d'autre part, à travers la notion de réseau sémantique, Picoche est amenée à prendre également en compte le lexique de moyenne fréquence.

En outre, son approche, en ancrant clairement l'étude du lexique sur un socle anthropologique, fournit une motivation puissante à l'étude lexicale :

« la spécificité d'une langue repose en grande partie sur la structure de ses polysèmes, qui sont en même temps des manières spécifiques de voir le monde. L'intérêt est grand, de présenter les différents sens du verbe abattre (concrets et abstrait), comme étroitement liés, parce que nous trouvons là une structure mentale de base, fondée sur la verticalité de l'espèce humaine, défi aux lois de la pesanteur, qui fait que l'homme debout est l'homme vivant, fort, bien portant, courageux, plein de la conscience de sa dignité, tandis que l'homme couché est mort, malade, fatigué, endormi, ou humilié. Il y a là une de ces métaphores fondamentales qui sont notre pain quotidien et dont il est de la plus haute importance de prendre conscience, un de ces passages du concret à l'abstrait dont nous aurons l'occasion de reparler sous le nom de « subduction». (Picoche, 1993).

En se démarquant de la tradition lexicologique (illustrée notamment autrefois par le Dictionnaire du Français Contemporain) qui préférait « dégrouper » le plus possible les acceptions, de manière à éviter tout risque de confusion, Picoche prend le parti d'un approche qui intègre la mémoire, l'histoire de la langue dans la compétence lexicale. Elle insiste sur la continuité plus que sur les ruptures, ne s'y résolvant que lorsque le fossé est trop grand entre les acceptions (par exemple dans le cas des deux sens d'abîmer : abîmer « détériorer » ou «jeter dans 
un abîme ») ? Du point de vue lexicographique, selon elle, il n'y a pas de risque à montrer la continuité des emplois dans un article de dictionnaire unique « bien structuré », tandis que cela présente l'avantage de « révéler l'ordre et l'intelligibilité sous ce qu'on aurait pu prendre pour du désordre et de l'absurdité ». Une telle approche, riche au plan culturel, n'est certes pas forcément la plus pertinente lorsqu'il s'agit, par exemple, de sélectionner le terme adéquat dans une optique de production de texte, ou même d'expliquer son sens au cours d'une lecture. Il faut la considérer comme ce qu'elle veut être : un moyen de mieux comprendre les liens qui se tissent entre les mots, d'accéder au potentiel symbolique du matériel lexical.

\subsection{Champ lexical et champ sémantique}

Les notions de champ lexical et de champ sémantique et aujourd'hui de réseau sémantique font partie de ces notions dont le caractère opératoire est discutable, mais dont il est difficile de se passer. Avant de préciser leur usage didactique, revenons sur quelques unes des utilisations qui en ont été faites en linguistique et en psycholinguistique. D’après Catherine Fuchs ${ }^{(10)}$ " on réserve généralement le terme champ sémantique pour caractériser le fonctionnement propre à une unité lexicale, et celui de champ lexical pour décrire des relations entre plusieurs unités lexicales ». Un champ sémantique rassemble les diverses significations d'un mot - polysémique - de la langue à un moment donné de son histoire, c'està-dire appréhendé en synchronie. Quant au champ lexical, il permet de regrouper les lexèmes qui entretiennent des relations sémantiques (synonymie, antonymie, hypo/hyperonymie).

Mel'čuk et al. (1995, p. 173-176), articulent, dans une perspective lexicographique, les notions de champ sémantique et champ lexical, en leur donnant un sens complémentaire. Ainsi, dans leur approche, un champ sémantique comporte «l'ensemble des lexies qui ont une même composante identificatrice de champ ». Par exemple, PLUIE, NEIGE, GRÊLE, BROUILLARD, TEMPÊTE et ORAGE comportent la même composante identificatrice 'phénomènes atmosphériques' parce que l'on peut extraire les renseignements lexicographiques communs à l'ensemble des membres du champ et les réunir dans l'article de dictionnaire consacré à la lexie identificatrice de champ. Le champ lexical, véritable objet du travail du lexicographe, est constitué de l'ensemble des vocables (i.e. des unités lexicales non désambiguïsées) dont les « lexies de base » ${ }^{(11)}$ appartiennent au même champ sémantique. D'un point de vue didactique, une telle conception peut servir de base à un travail fort utile sur la définition.

Cependant, il est frappant de constater que l'usage didactique des notions de champ lexical et de champ sémantique apparaît en définitive assez peu dans les séances dédiées spécifiquement à l'apprentissage lexical, mais bien plutôt au cours des séances de lecture et d'explication de texte. Jouili (2007), observe que sous ces rubriques, c'est bien l'approche thématique traditionnelle du lexique qui est envisagée dans les Instructions Officielles du français pour le collège

(10) Dans 1'article « champ sémantique et champ lexical» de 1'Encyclopaedia Universalis.

(11) La « lexie de base » est la lexie à partir de laquelle peut se construire l'ensemble des définitions des autres lexies (pour une définition plus précise, ouvr. cit., p. 159). 
[I.O. 05] et pratiquée dans les manuels. La démarche préconisée consiste en effet « à regrouper les mots d'un champ lexical autour d'un même thème (la montagne, la ville, l'amitié, la colère, etc.). Ce travail, selon les I.O., " doit être mené en relation avec la lecture et l'observation des textes, lorsqu' on y explore un champ sémantique : il passe alors par une activité de recherche qui amène à effectuer des relevés, à établir des réseaux (isotopies) et à les analyser. " [I.O. pp. 127-128]. En superposant lexique et thème, on se prive à la fois d'une analyse plus fine de la dimension linguistique, à travers la syntaxe et le fait collocatif, ainsi que d'une appréhension stylistique ou littéraire, puisque le texte, dans bien des cas, est réduit, au moins en première approche, à un ensemble de mots clés, les exercices proposés se faisant ensuite à partir de cette liste de termes extraits, sans que soit véritablement pensée leur intégration au texte. La notion d'isotopie peut certes donner le change, mais d'une part elle est rarement utilisée dans son sens technique, et d'autre part, sa limite théorique même la condamne à méconnaître le fait syntaxique. Autrement dit, il faut reconnaître que ces notions servent avant tout d'alibi techniciste et ne permettent pas un traitement rigoureux des faits observés, sans favoriser pour autant une meilleure compréhension littéraire.

\subsection{Réseaux sémantiques}

En psycholinguistique, la notion de réseau sémantique peut être liée en premier lieu à la notion de lexique mental. Même si, de manière très simplificatrice, le lexique mental ${ }^{(12)}$ peut être appréhendé comme une « liste » d'unités lexicales auxquelles seraient associées les informations nécessaires à une utilisation appropriée (Pillon, 1993, p. 13), cette conception n'implique pas une vue statique. Le matériau lexical n'est pas stocké en mémoire comme un empilement de mots, il est activé de manière dynamique ; l'organisation du lexique mental ne se superpose pas à l'organisation du lexique telle qu'elle est consignée par les linguistes ou les lexicographes. Plutôt que comme un «stock» ou un « trésor » semblable à un dictionnaire, on préfère aujourd'hui se représenter ce lexique comme le résultat, toujours évolutif, de processus créant des liens sémantiques nouveaux. En effet, ce qui le caractérise c'est sa souplesse et sa plasticité ; des liens inédits sont capables d'être trouvés par le sujet en fonction de ses intérêts propres. Le lexique mental est organisé à partir du sens plutôt que de la forme des mots, mais la parenté morphologique lorsqu'elle est associée au sens, joue un rôle important pour faciliter les associations et la mémorisation. L'analyse compositionnelle des unités lexicales semble jouer également un rôle au moins pour le traitement de certains mots complexes, qui pourraient être récupérés par une procédure compositionnelle (Pillon, 1993, p. 83), les recherches semblant montrer cependant qu' on a affaire à un double codage, l'un permettant l'accès direct, l'autre empruntant la voie compositionnelle.

Cependant, les réseaux sémantiques ne sont pas seulement individuels, mais aussi, pour une large part socialisés, notamment à travers la manière dont est appris le système de la langue. Si les liens non prévisibles sont fréquents, chaque sujet découvre aussi, empiriquement ou de manière plus guidée, les logiques in-

(12) Sur cette notion, voir par exemple Pillon (1993) et Bobin (1998). 
ternes qui organisent les séries lexicales et les règles qui gouvernent la composition des mots dans sa propre langue en fonction de patterns hautement spécifiques ; l'apprentissage, scolaire ou extra-scolaire, s'appuie aussi sur les outils de la «grammatisation » propres aux sociétés de l'écrit (Auroux, 1992), outils qui constituent un accélérateur formidable pour la prise de conscience linguistique. Dans l'apprentissage lexical, la question des outils (dictionnaires, et aujourd'hui sites web), joue donc un rôle fondamental.

La notion de réseau, elle a été utilisée de différentes façons dans une perspective didactique :

- comme moyen de structurer la réflexion linguistique : c'est le cas des réseaux sémantiques de Picoche (voir 2.1.3), constitués à partir de mots de haute fréquence, des caractéristiques sémantiques de leurs actants, des associés moins fréquents ; c'est le cas également dans l'approche de la lexicologie combinatoire, qui distingue deux manières de connecter les unités lexicales : les liens paradigmatiques («dérivation sémantique ») le font grâce à des relations sémantiques qui n'ont pas forcément une correspondance morphologique; les liens syntagmatiques traduisent les collocations contrôlées par les unités lexicales (ex. contenir sa joie, colère noire...); - sur un plan plus pratique, et en lien avec l'approche précédente, comme moyen de faciliter l'accès aux données lexicales, par exemple à travers un site lexical « qui vise à modéliser la connaissance lexicale comme un réseau d'entités interconnectées (Jousse, Polguère, Tremblay, 2008) ;

- enfin, en lien avec la lecture-écriture, comme un moyen de personnaliser l'appropriation du lexique par l'apprenant, qui note de manière personnelle les associations sémantiques telles qu'il les perçoit au fur et à mesure de ses lectures ou de ses productions. Une démarche allant dans ce sens est proposée dans Elalouf et Keraven (2004) à travers l'appropriation d'un champ onomasiologique (par exemple, pour aider à construire le concept de chevalier et être en mesure de mobiliser et de structurer le champ lexical correspondant : l'appui sur la documentation et l'écriture de textes permet la construction de l'univers de référence en stabilisant les connaissances historiques des élèves, tout en attirant leur attention sur le matériau lexical et en aboutissant à un classement des faits lexicaux.

\subsection{Syntagmatique, combinatoire lexicale, phraséologie}

Un certain nombre de courants linguistiques, très différents, mais convergents sur certains points importants (notamment dans leur refus d'entériner la coupure entre lexique et grammaire) ont contribué ces dernières années à renouveler en profondeur l'approche de la phraséologie et de l'idiomaticité ${ }^{(13)}$, phénomènes traditionnellement considérés comme faisant exception au système de

(13) On renverra en particulier aux travaux connus sous le nom de " grammaires de construction » ainsi qu'à ceux des contextualistes britanniques (pour une comparaison, voir Legallois et François, 2006). Pour un « radical» comme Hoey (2005) chaque lexème est « amorcé », pour les locuteurs dans un certain co-texte et contexte (notamment collocationnel, mais également propre au genre discursif), et c'est cet amorçage qui conditionne ensuite l'usage qui peut en être fait. 
la langue. Un des apports principaux de ces nouvelles approches pour la didactique du lexique a été de contribuer au décloisonnement du mot, de manière à prendre en compte, de manière beaucoup plus systématique, les «blocs phraséologiques » et autres associations lexicales (collocations).

Les grammaires de constructions et, plus spécifiquement, les « grammaires émergentes » (Hopper, 1998), ont suscité l'intérêt des acquisitionnistes, parce qu'elles peuvent s'inscrire aisément dans la perspective du développement. Pour les didacticiens, les contributions essentielles restent celles des contextualistes ${ }^{(14)}$, appuyés sur la longue tradition des corpus en Grande-Bretagne, et plus encore celles issue de la lexicologie combinatoire.

L'introduction des corpus - et d'outils complémentaires, comme les concordanciers - fournit en effet une base empirique à l'observation et à l'étude des phénomènes lexicaux, ce qui explique l'intérêt didactique qu'elle peut présenter ; il reste cependant un gros travail à réaliser, notamment en France, pour faciliter l'accès aux corpus, et surtout pour permettre leur exploitation dans les classes.

L'intérêt de la lexicologie explicative et combinatoire est tout différent : elle a permis - en premier lieu, à travers la notion de fonction lexicale ${ }^{(15)}-$, d'introduire des types de relation sémantique jusque là ignorés ou méconnus dans le cadre de la didactique du lexique; en second lieu, la théorie sens-texte dont elle est issue, plaçant au cœur de son système la notion de paraphrase ${ }^{(16)}$, fournit naturellement, à travers les outils qu'elle produit, des manières diverses et complémentaires d'encoder les relations sémantiques, tout en décrivant de manière précise la plupart des types de collocation.

Du point de vue didactique, Chanfrault-Duchet (2004, p. 103), considère que le tournant syntagmatique est rendu particulièrement nécessaire en raison des difficultés que rencontrent massivement aujourd'hui les élèves dans leurs productions écrites dans le domaine de ce qu'il est convenu d'appeler d'un terme vague les «maladresses d'expression » ${ }^{(17)}$ : les exemples fournis, issus d'une classe de $3^{\mathrm{e}}$, se situent sur plusieurs plans, je me limiterai ici à la question des collocations ( $c f$. l'exemple fourni : le poète pousse une analyse approfondie des personnages). De telles erreurs lexicales peuvent être analysées à différents niveaux : connaissance insuffisante des contraintes stylistiques d'un genre scolaire ; méconnaissance du collocatif verbal que doit sélectionner, etc. Pour l'auteur, il s'agit de fonder une approche lexicale « à la française », inspirée de l'approche syntagmatique du lexique, inspirée des travaux des lexicalistes britanniques de la lexical approach (Lewis, 1993, 1997). L'auteur explique la difficulté à faire passer l'idée d'un travail sur les constructions préfabriquées auprès de professeurs de lettres, par le fait que ceux-ci privilégient la créativité, au détriment des formes pré-construites. Cette remarque soulève un problème intéressant : les collocations et les locutions, lorsqu'elles ne correspondent pas à la for-

(14) Pour une présentation de ce courant, voir Williams (2003).

(15) Pour une présentation de cette notion, voir par exemple voir Mel'čuk et al. (1995).

(16) Polguère (2004) illustre les vertus de la paraphrase comme outil pédagogique de modélisation des liens lexicaux.

(17) On peut contester cette analyse un peu catastrophiste, mais cela n'obère pas l'intérêt de la démarche proposée. 
mulation la plus courante, comme c'est souvent le cas des collocations formées avec des verbes-supports (ex. prendre le train), sont marquées stylistiquement, et il est indispensable d'intégrer les normes d'usage, si l'on veut qu'elles puissent être mobilisées de manière pertinente : une collocation permettant de marquer l'intensité comme blême comme le linge est stylistiquement marquée ; une locution comme pousser le dernier soupir est un cliché qui ne peut pas être utilisé dans n'importe quel contexte en place de mourir. Une stylistique des genres est donc indissociable de tout travail portant sur la phraséologie et le figement.

\section{Vers une meilleure intégration des apprentissages lexicaux}

Le point de vue défendu ici, rappelons-le, est qu'il n'y a pas à choisir entre, d'une part, une approche visant le développement de la conscience du système lexical et une approche intégrée, mais qu'il s'agit de délimiter plus exactement leur complémentarité. Nous pensons qu'il est nécessaire qu'un petit nombre de notions permettant une meilleure compréhension du système lexical soient bien assimilées par les élèves. Mais il est également utile de préciser le type d'activité qu'il est possible de concevoir en lien avec les activités d'expression et de communication.

\subsection{Le lien avec les activités de lecture}

Au cours de la lecture, une fréquence même relativement faible de " mots inconnus » ${ }^{(18)}$ suffit à compliquer le décodage et à entraver la compréhension (Carver, 1994) ; mais l'activité de lecture représente en elle-même un élément important pour l'acquisition lexicale ; les recherches ont montré qu'il est nécessaire que le lecteur rencontre un lexème à de nombreuses reprises, et dans différents contextes pour construire progressivement son sens (Nagy et al., 1985, $1987 \mathrm{a}$ et b). La lecture fournit une autre porte d'entrée au lexique : comme le remarque David (2000), au cours de l'apprentissage de la lecture le lexique oral est reconfiguré par l'acquisition des formes écrites, notamment dans sa dimension morphologique ; il y a aussi une interaction évidente entre l'appropriation de certains genres propres à l'écrit et le développement des répertoires lexicaux.

D'un point de vue didactique, le lien entre lexique et lecture-écriture s'effectue différemment suivant les niveaux. A l'école maternelle (et parfois au-delà), la lecture d'albums et d'histoires joue un rôle important; les formes de médiation utilisées par le lecteur adulte permettent l'accès à un lexique nouveau et peu familier. Dans le courant de la séance de lecture, et ce de l'école primaire au lycée, des explicitations lexicales sont généralement effectuées mais elles fournissent rarement la base d'un travail ultérieur sur le lexique ${ }^{(19)}$. Par définition, ces explicitations, dont il ne s'agit pas de contester la nécessité, ne peuvent être que rapides, de manière à ne pas rompre l'intérêt du texte. Picoche et Souhaite (2004, p. 216) tout en signalant à juste titre que leur proposition ne peut fonctionner pour tous les textes donnent l'exemple d'un renversement intéressant de la

(18) Nous reprenons ce terme, souvent utilisé dans ce contexte, malgré ses ambiguïtés.

(19) Pour des propositions en ce sens dès le cours préparatoire, voir Gomila, 2004. 
problématique : ils suggèrent une «étude en réseau », à partir de l'exemple de la fable Le loup et l'agneau de La Fontaine, étudiée à partir de l'article RAISON du Dictionnaire du français usuel : en se fondant sur le sens des termes mobilisés dans l'article de dictionnaire (DÉDUIRE, CONCLURE, INDUIRE, DÉMONTRER, ÉTAYER, OPINION, etc.), on peut explorer sa mise en œuvre textuelle. Il s'agit, au terme de l'analyse, de prendre conscience de la polysémie de RAISON, « son contenu pouvant être envisagé comme pur et simple mécanisme logique, ou comme ce même mécanisme, mis au service d'intérêts et de passions » (ouvr. cit. p. 217).

Se situant dans une optique très différente, Chabanne et al. (2008) mettent en avant l'apport que représente l'expérience littéraire pour la compréhension lexicale. Par exemple, le sens de banni gagne à être appréhendé de prime abord à travers la mise en scène qui en est faite dans un album, cette lecture fournissant un script remobilisable. Est favorisée également l'appropriation par les élèves euxmêmes des éléments lexicaux : on leur demande de lister les mots qu'ils aiment, ceux qu'ils n'aiment pas, ceux qu'ils ne comprennent pas, etc. Le risque d'une telle approche pourrait être de revenir à l'unité isolée, mais les auteurs remarquent que les élèves choisissent fréquemment des syntagmes (remarquons qu'il s'agit souvent de collocations), telles que (bronze noirci, murs verdis, terrible secret, passion folle, rire inhumain, voire des énoncés mettant en jeu la structure actancielle (donne-moi le miroir). Nous nuancerons donc les conclusions des auteurs, qui récusent le clivage opposant enseignement incident et enseignement systématique : on voit bien, à travers les exemples qu'ils fournissent comment peut se combiner l'approche « intégrée » et l'approche «systématique », puisque les concepts acquis lors des séances spécifiques consacrées à la construction des notions lexicales de base peuvent être facilement réinvestis lors des séances articulées sur la lecture ou l'écriture ; inversement, il est également possible de partir des observations des élèves lors de ces séances de lectures littéraires pour les aider à construire ensuite les notions fondamentales dont ils auront besoin.

\subsection{Ecriture et production d'écrits}

Les mêmes remarques peuvent être formulées à propos des activités d'écriture. Masseron (2008), et Grossmann et al. (2008) fournissent deux exemples complémentaires de la manière dont peut être favorisée l'intégration du lexique des émotions au cours d'un projet d'écriture narrative. Caroline Masseron, qui vise l'appropriation par les élèves du lexique de la peur, dans une classe de seconde, fait l'hypothèse que quatre opérations (conception-invention du texte, macro-organisation et planification, mise en mot et mise en texte, révision permettent chacune d'envisager la mobilisation lexicale de manière spécifique. La démarche mise en œuvre permet donc de guider les apprenants en les conduisant d'une réflexion sur les représentations et les expériences de la peur, à travers leur lexicalisation, à une recherche des effets à produire sur le lecteur. Les différentes structures syntaxiques (résultatives, causatives, pronominales...) sont également prises en compte. Grossmann et al. (2008) proposent quant à eux, pour des classes de primaire et de collèges, une série de parcours : le premier part du lexique lui-même pour déclencher l'écriture, tandis que les suivants s'appuient sur les problèmes posés par la mise en scène des émotions et des sentiments dans le texte pour intégrer les aspects lexicaux : il s'agit en particulier de pouvoir décrire 
les sentiment d'un personnage en montrant en quoi ils sont reliés à la dynamique narrative elle-même. Les collocations sont abordées notamment à partir de la dimension aspectuelle, qui permet de montrer la mobilité et l'évolutivité des sentiments. Enfin, un travail est prévu sur la construction détachée, qui permet d'intégrer la caractérisation lexicale des sentiments au texte. D'autres recherches insistent sur la productivité d'un travail préalable de catégorisation : dans une expérimentation menée au cours moyen 1 et 2 (Duvignau et Garcia-Debanc, 2008), les élèves ont été amenés à classer des verbes fréquents en fonction de critères sémantiques. Il apparaît que ce type de tâche permet ensuite une meilleure mobilisation de la palette lexicale lors des travaux d'écriture.

\section{Conclusion}

Cet article visait essentiellement à montrer qu'il est temps de dépasser un certain nombre de faux dilemmes, pour construire, sur la durée, sinon un programme, du moins des principes permettant le développement de l'apprentissage lexical. Rappelons pour finir quelques uns des éléments qui pourraient en constituer les principales « briques » constitutives :

- pour chaque niveau d'enseignement, une meilleure spécification des répertoires lexicaux à privilégier, en fonction des genres scolaires et non scolaires mobilisés (ex. le lexique des sensations et des sentiments peut s'inscrire comme un objectif utile à la fin de la scolarité primaire et au début du collège, en lien avec l'étude de récits et de romans; le lexique spécifique du genre dissertatif est à travailler plus spécifiquement à partir de la seconde, etc.) ;

- pour l'ensemble de la scolarité primaire et secondaire, définir le socle des notions nécessaires au volet «structuré » de l'apprentissage, volet qui est indispensable; les propositions de la lexicologie explicative et combinatoire ont, sur ce plan, le mérite de la cohérence et permettent d'intégrer de manière satisfaisante la dimension syntagmatique, dont la prise en compte permettra de poursuivre le nécessaire «décloisonnement» du lexique et son intégration pleine et entière aux activités de langue ;

- établir une meilleure connexion avec l'enseignement des notions grammaticales et mettre en relation des phénomènes aujourd'hui présentés comme disjoints ( $c f$. la dérivation, dans l'enseignement du lexique, et la flexion dans l'enseignement de la grammaire);

- développer le lien entre lexique et discours à travers la question des usages et des normes d'une part; à travers la question des spécificités génériques d'autre part; il reste en particulier du travail, y compris dans les outils proposés par les lexicologues, pour prendre sérieusement en compte la question des usages discursifs du lexique ;

- enfin, mieux cerner les articulations permettant de relier concrètement certains objectifs lexicaux et certains problèmes de lecture ou d'écriture.

Complémentairement, doivent encore être mieux documentées les connaissances lexicales des élèves aux différents niveaux de la scolarité. Il ne m'a pas été possible, dans le cadre de cet article de développer la question de la progression : comme Halté et Rondelli (2010), je pense qu'il est possible de concilier 
une «progression non strictement linéaire et un principe de progressivité » des apprentissages lexicaux, en ciblant des objectifs précis, tout en laissant des marges de manœuvre en fonction des besoins pédagogiques. Un programme d'acquisition des notions métalinguistiques de base est nécessaire et doit être consolidé sur la durée : une notion comme la polysémie, comme le rappelle Nonnon (2008, 63-64), est présente dans les programmes de français à tous les niveaux, presque exactement dans les mêmes termes, sans qu'aucun développement curriculaire susceptible de différencier le travail à mener ne soit proposé. Mieux prendre en compte cet enjeu curriculaire est sans doute, l'une des conditions du renouvellement de la didactique du lexique pour ces prochaines années ${ }^{(20)}$.

\section{Bibliographie}

AurouX, S. (1992) : «Le processus de grammatisation et ses enjeux », Histoire des idées linguistiques, t. II, Liège, Mardaga, 11-64.

BABIN, J.-P. (1998) : Lexique mental et morphologie lexicale, Berne, Peter Lang.

CALAQUE, E. (2002) : Les Mots en jeu. L'enseignement du vocabulaire, CRDP de Grenoble.

Calaque, E. et David, J. (2004) : Didactique du lexique. Contexte, démarches, supports, Bruxelles, De Boeck Université, pp. 103-125.

CAPPEAU, P. (2008) : « La recherche du mot juste à l'oral », in Grossmann, F. et Plane, S., Lexique et production verbale. Vers une meilleure intégration des apprentissages lexicaux, Lille, Presses Universitaires du Septentrion, pp. 71-83.

CARVER, R.P. (1994) : " Percentage of unknown vocabulary Words in Text as a Function of the relative Difficulty of the Text : Implications for Instruction », Journal of Reading Behavior, 26, 413-43.

CHANFRAULT-DUCHET (2004) : «Vers une approche syntagmatique du lexique en didactique du français ", in Calaque, E. et David. J., Didactique du lexique, Contextes, démarches, supports, Bruxelles, De Boeck, pp. 103-114.

CHARLES, F. (2008): Du mot au «mot juste», conceptions et représentations dans l'enseignement/apprentissage du lexique au cycle 3 de l'école élémentaire, Thèse sous la direction de Sylvie Plane, Université Paris V.

CotTeZ, H. (1977) : « Enseignement et faits lexicaux », Langue française, 33, 7989.

(20) Je remercie vivement Françoise Wirth et Alain Polguère de leur relecture et de leurs suggestions. Les imperfections ou erreurs qui demeurent sont de mon seul fait. 
DELESALLE, S. (1975) : «La recherche du "mot juste", son sens et son rôle », Langue française, 26, 30-40.

De Mauro, T (1983) : « Il vocabolario di base », Prometeo, 3, 127-31.

DUVignaU, K. (2005) : «Pour un apprentissage-enseignement du lexique verbal calqué sur l'acquisition : revisite et apport des "métaphores/erreurs"》 des enfants de 2 à 4 ans », in Grossmann F., Paveau, M.-A. et Petit, G., Didactique du Lexique : langue, cognition, discours, Grenoble, ELLUG, pp. 37-49.

Elalouf, M.-L. \& KeRAVEN, J. (2004) : «L'acquisition du lexique à l'épreuve d'un grand corpus de textes d'élèves ", in Calaque, E. et David. J., Didactique du lexique, Contextes, démarches, supports, Bruxelles, De Boeck, pp. 185-197.

GomiLA, C. (2004) : «Le traitement du lexique dans la leçon de lecture », in Calaque, E. et David, J., Didactique du lexique, Contextes, démarches, supports, Bruxelles, De Boeck, pp. 157-170.

Gougenheim, G., MicheA, R., Rivenc, P. \& SAuvageot, A. (1956) : L'élaboration du français élémentaire, étude sur l'établissement d'un vocabulaire et d'une grammaire de base, Didier, Paris. (Nouv. éd. refondue et augmentée sous le titre L'élaboration du français fondamental : étude sur l'établissement d'un vocabulaire et d'une grammaire de base, Didier, Paris, 1964).

Grossmann, F., Boch, F., CaVAlla, C. (2008) : «Quand l'écriture n'empêche pas les sentiments... Quelques propositions pour intégrer le lexique des sentiments dans la production de textes ", in Grossmann, F. et Plane, S., Lexique et production verbale. Vers une meilleure intégration des apprentissages lexicaux, Lille, Presses Universitaires du Septentrion, pp. 191-218.

Grossmann, F. \& Plane, S. (2008) : Lexique et production verbale. Vers une meilleure intégration des apprentissages lexicaux, Lille, Presses Universitaires du Septentrion.

GUERNIER, M.-C. (2005) : « Le lexique : apprentissage et enseignement », in Grossmann F., Paveau, M.-A. et Petit, G. (2005), Didactique du Lexique : langue, cognition, discours, Grenoble, ELLUG, pp. 247-265.

Halté, A. et Rondelli, F. (2010) : «Comment concilier une progression non strictement linéaire et un principe de progressivité des apprentissages en vocabulaire ? », Repères, 41, 125-146.

HOPPER, P. J. (1998) : «Emergent grammar», in M. Tomasello (Ed.), The new psychology of language : Cognitive and functional approaches, Mahwah : NJ : Lawrence Erlbaum Associates.

JOUILI, E. (2007) : « Sur la notion de champ lexical : de la norme lexicale à la variation lexicale en didactique du lexique », Rencontres Jeunes Chercheurs Normes, variations, identité, altérité, ED 268 Université Paris III, le 12 mai 2007, Consultable en ligne http://hal.archives-ouvertes.fr/ docs/00/23/94/54/PDF/JOUILI_actes_RJC2007.pdf.

LEEMAN, D. (2000) : «Le vertige de l'infini ou la difficulté de didactiser le lexique », Le français aujourd'hui, 131, 42-52.

LE FUR, D. (ss la dir. de) (2007) : Dictionnaire des combinaisons de mots, Paris, Le Robert.

LEGAllois, D. et FRANÇOIS, J. (2006) (dir.) : « Autour des grammaires de construction et de patterns ", Cahiers du Crisco. 
LEPOIRE-DUC, S. (2004) : «Interactions langagières et enjeux lexicaux », in Calaque E., David J., Didactique du lexique Contextes, démarches, supports, De Boeck, Bruxelles, pp. 143-156.

Lete, B., Sprenger-Charolles, L., \& Cole, P. (2004) : « MAnuleX : A gradelevel lexical database from French elementary-school readers », Behavior Research Methods, Instruments, \& Computers, 36, 156-166.

LEWIS, M. (1993) : The Lexical Approach: The State of ELT and a Way Forward, Hove, UK, Language Teaching Publications.

- (1997); Implementing the lexical approach: Putting theory into practice, Hove, UK, Language Teaching Publications.

LÜDI, G. (1997) : «Apprendre à construire des mots : la suffixation nominale chez des apprenants germanophones du français », Basle Electronic Working Papers, Linguistics, 1, University of Basel.

MARTINOT, C. (2006) : «Comment une pratique langagière naturelle peut être sollicitée dans l'apprentissage/acquisition du lexique en contexte scolaire », Mélanges CRAPEL, 29, 29-39.

MASSERON, C. (2008) : «Pour une topique de la peur : aspects psychologiques, sémiotiques, linguistiques ", in Grossmann, F. et Plane, S., Lexique et production verbale. Vers une meilleure intégration des apprentissages lexicaux, Lille, Presses Universitaires du Septentrion, pp. 161-190.

MATTHEY, M. (1992) : «L'obsession des mots. Les archives Reichenbach et la problématique des vocabulaires de base dans l'enseignement du français langue maternelle », Tranel, 18, 103-192.

Mel'ČUK, I., Clas, A. \& POlguere, A. (1995) : Introduction à la lexicologie explicative et combinatoire, Paris, Duculot.

MEL'ČUK, I. \& Polguère, A. (2007) : Lexique actif du français, Louvain la Neuve : De Boeck.

NAGY, W., HERMAn, P., ANDERSON, R. (1985) : «Learning words from context », Reading Research Quarterly, 20, 223-253.

NAGY, W., \& HERMAN, P. (1987) : « Breadth and depth of vocabulary knowledge : Implications for acquisition and instruction », in M. McKeown \& M. E. Curtis (Eds.), The Nature of Vocabulary Acquisition (Vol. 19-35). Hillsdale, NJ : Lawrence Erlbaum.

NAGy, W. E., Anderson, R. C., HERMAN, P. A. (1987) : «Learning word meaning from context during normal Reading », American Educational Research Journal, 24(2), 237-270.

NONNON, E. (2008) : «Apprendre des mots, construire des significations : la notion de polysémie à l'épreuve de la transdisciplinarité », in Grossmann, F. et Plane, S., Lexique et production verbale. Vers une meilleure intégration des apprentissages lexicaux, Lille, Presses Universitaires du Septentrion, pp. 43-69.

OGDEN, C. K. (1934) : The system of Basic English, Harcourt Brace and Co, New York.

PAVEAU, M.-A. (2000) : «La "richesse lexicale", entre apprentissage et acculturation », Le Français Aujourd'hui, 131, 19-30.

Petit, G. (2000) : «Didactique du lexique et problématique de l'unité lexicale : état d'une confusion », Le Français Aujourd'hui, 131, 53-62. 
PLANE, S. (2004) : «Un consensus à interroger : la question des "mots difficiles". Quelques éléments d'une enquête sur le repérage des problèmes lexicaux », Actes du $9^{\mathrm{e}}$ colloque de l'AIRDF, Québec, 26 au 28 août 2004, en ligne www.colloqueairdf.fse.ulaval.ca/ fichier/.../ sylvie-plane.pdf, consulté le $11 / 03 / 2011$

Picoche, J. (1993) : Didactique du lexique, Paris, Nathan.

- (1977, rééd. 1992) : Précis de lexicologie française. L'étude et l'enseignement $d u$ vocabulaire, Paris, Nathan.

Picoche, J. et Rolland (2002) : Dictionnaire du français usuel, Paris, Duculot.

Picoche, J. et Souhaite, S. (2004) : «L'utilisation du Dictionnaire du français usuel pour l'enseignement du vocabulaire », in Calaque, E. et David, J., Didactique du lexique. Contexte, démarches, supports, Bruxelles, De Boeck Université, pp. 215-225,

PILlon, A. (1993) : La mémoire des mots. Ses unités, son organisation, Mardaga, Psychologie et sciences humaines.

POlguÈre, A. (2004) : «La paraphrase comme outil pédagogique de modélisation des liens lexicaux », in Calaque, E. et David, J., Didactique du lexique. Contexte, démarches, supports, Bruxelles, De Boeck Université, pp. 115125.

Rey-Debove, J. (2004) : Le Robert Brio, Paris, Editions Le Robert.

RINCK, F. (2006) : «L'article de recherche en Sciences du langage et en Lettres. Figure de l'auteur et identité disciplinaire du genre », thèse en Sciences du Langage, Grenoble III.

Tomasello, M. (2003) : Constructing a Language : A Usage-Based Theory of Language Acquisition, Harvard University Press.

TREMBLAY, O. (2004) : «Pour un apprentissage structuré de l'enseignement-apprentissage du lexique », in Calaque, E. et David, J., Didactique du lexique. Contexte, démarches, supports, Bruxelles, De Boeck Université.

- (2009) : Une ontologie des savoirs lexicologiques pour l'élaboration d'un module de cours en didactique du lexique, Thèse présentée à la Faculté des études supérieures en vue de l'obtention du grade de PhD en Didactique, Université de Montréal.

VigneR, G. (1993) : «Le monde, les mots, l'école. Eléments d'une didactique du vocabulaire à l'école élémentaire ", Repères, 8, 191-209.

- (1997) : «Culture, connaissance du monde et lexique : le rôle des schémas de contenus dans l'acquisition du vocabulaire », Triangle, 16, 41-72.

Williams, G. (2003) : «Les collocations et l'école contextualiste », in Grossmann F. et Tutin A. (dir.), Collocations : analyse et traitement, Travaux et recherches en linguistique appliquée, De Werelt, pp. 33-44. 